\title{
PERCEPTION DES VOYELLES NASALES DU FRANÇAIS PAR LES ÉTUDIANTS SERBES DE FLE
}

\author{
UDC 811.133.1'342.4-057.875(497.11)
}

\section{Nataša Ignjatović}

Université de Niš, Faculté de philosophie, Département de langue et littérature françaises, Serbie

Résumé. Dans cet article nous analysons la perception des voyelles nasales du français par les étudiants serbes de FLE, les phonèmes qui n'existent pas dans le système vocalique de la langue serbe et de ce fait représentent souvent les difficultés de perception et de production aux apprenants serbophones. Étant donné que le système perceptif de chaque individu se forme lors de l'acquisition de la langue maternelle, l'oreille n'est pas habituée à entendre les phonèmes qui n'existent pas dans la langue maternelle des apprenants, ce qui entraîne les erreurs de perception pendant l'appropriation des sons d'une langue étrangère (Trubetzkoy [1938]2005 ; Best 1994 ; Kuhl 2000). Les résultats de la présente recherche montrent que les étudiants serbes de FLE perçoivent et distinguent avec réussite le trait oralitélnasalité, tandis qu'ils tendent à remplacer une voyelle nasale par l'autre. Au total, la plus grande réussite est éprouvée dans

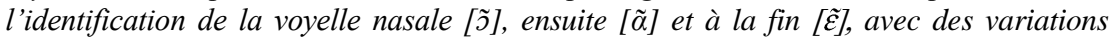
selon la position de la voyelle dans le mot ou selon le groupe d'étudiants interrogé.

Mots-clés : les voyelles nasales, la perception, la langue française, les étudiants serbes, l'acquisition.

\section{INTRODUCTION}

Quand on pose la question aux étudiants en première année d'études, au premier cours de phonétique et phonologie, sur le nombre des voyelles nasales du système vocalique de la langue française, ils hésitent d'abord et puis, le plus souvent, ils répondent : «deux : [õ] et $[\tilde{\alpha}] \gg$. Il nous semble, alors, que pendant l'enseignement scolaire ils s'approprient les voyelles nasales intuitivement, en les assimilant et en les classant dans leur système de sons existants, sans vraiment en prendre conscience en faisant des activités qui visent l'acquisition de quatre

Submitted July 2, 2019; Accepted September 15, 2019

Corresponding author: Nataša Ignjatović

University of Niš, Faculty of Philosophy

E-mail: natachamina08@gmail.com 
voyelles nasales du français standard $[\tilde{\varepsilon}$, õ, $\tilde{\alpha}$, ̃̃,], et sans référence aux graphèmes correspondantes. C'est la raison pour laquelle nous voudrions vérifier dans ce travail s'ils sont capables de distinguer les voyelles orales/nasales et de percevoir correctement les voyelles nasales.

Les difficultés de perception et de prononciation des sons lors de l'apprentissage d'une langue étrangère sont attribuées le plus souvent aux différences entre les systèmes phonologiques de la langue maternelle et la langue étrangère. "On n'entend et on ne reconnaît que ce qu'on a l'habitude d'entendre et de reconnaitre » (Lhote 1995, 28, cité dans Cornaire 1998, 113). C'est-à-dire que si certains sons ne figurent pas dans le répertoire de notre langue maternelle ils sont, par défaut, difficiles à repérer. Selon Trubetzkoy, nous percevons les sons à travers un crible phonologique ce qui nous permet de ne distinguer que les sons connus, auxquels on était habitués lors de l'acquisition de la langue maternelle, tandis les sons de la langue étrangère, passés par le filtre de la langue maternelle, sont mal décodés (Trubetzkoy [1938] 2005, 38).

La théorie d'aimants perceptifs - 'perceptual magnets theory', explique les étapes de l'acquisition des sons chez les enfants : les nourrissons sont capables de percevoir et discriminer les sons de n'importe quelle langue qu'ils écoutent dans leur entourage et cette caractéristique est universelle. Mais, dès le sixième mois, ils commencent à sauvegarder les sons de leur langue maternelle, en créent des cartes mentales avec les prototypes des sons. À partir de ce moment-là, leur capacité à discriminer les sons des langues étrangères commence à diminuer. À 12 mois, ils classifient les sons entendus dans les prototypes qui fonctionnent comme aimants, c'est à dire qu'ils attirent les sons proches du prototype. Ainsi, l'apprentissage d'une langue étrangère et des nouveaux sons s'avère difficile, car les sons sont repérés et classifiés à travers le système des prototypes, propre à la langue maternelle (Kuhl 2000).

Selon le modèle d'assimilation perceptive - 'perceptual assimilation model', la perception des sons d'une langue étrangère dépend des ressemblances et différences articulatoires et acoustiques qui existent entre la langue maternelle et la langue étrangère (Best 1994). D'un côté, l'auditeur va assimiler les sons de la langue étrangère à la catégorie existante dans sa langue maternelle, s'il les perçoit comme similaires, en remarquant pourtant qu'il ne s'agit pas de sons identiques. De l'autre côté, s'il n'y a pas de ressemblances articulatoires entre les sons de la langue maternelle et la langue étrangère, l'apprenant va percevoir les différences, et ne pourra pas assimiler les sons de la langue étrangère dans le système de sa langue maternelle (Best 1994). L'auteure propose alors quatre modèles possibles d'assimilation perceptive : 1 . deux phonèmes différents d'une langue étrangère peuvent être assimilés à deux catégories de la langue maternelle ; 2 . deux phonèmes d'une langue étrangère peuvent être assimilés à une seule catégorie de la langue maternelle ; 3 . deux phonèmes différents d'une langue étrangère dont l'un ressemble plus au phonème de la langue maternelle, peuvent être assimilés à une seule catégorie de la langue maternelle ; 4. si l'articulation des phonèmes d'une langue étrangère est différente de celle de la langue maternelle, les phonèmes ne peuvent pas être perçus, et donc ils ne sont pas assimilés (Best 1994, 14-15).

D'après le modèle d'acquisition de la parole de Flege - 'Speech Learning Model', deux mécanismes se rapportent à l'acquisition des phonèmes : l'assimilation et la dissimilation des catégories phonétiques. La personne qui apprend une langue étrangère, s'approprie une nouvelle catégorie phonétique, différente d'une catégorie existante dans sa langue maternelle, et dans ce cas-là, la catégorie da la langue maternelle va être différente de la 
même catégorie existante chez celui qui n'apprend pas de langues étrangères. C'est à dire que lorsqu'on apprend une nouvelle langue, les catégories phonétiques existantes dans la langue maternelle sont susceptibles de changer (Flege 2003, 330). En ce qui concerne les possibilités d'acquisition d'une nouvelle langue, Flege considère que les capacités se rapportant à l'acquisition des catégories phonétiques pour les voyelles et consonnes diminuent avec l'âge (Flege 2003, 328). Par contre, Lauret constate une différence dans l'acquisition de la parole par les apprenants moins âgés d'une part, et les apprenants plus âgés d'autre part, en disant que ces derniers « tendent à traiter le signal L2 linguistiquement » tandis que les élèves moins âgés le font «auditivement sans référence à des traits linguistiques déjà développés » (Lauret 2007, 26-27). Cela veut dire que les apprenants plus âgés n'ont pas nécessairement moins de succès dans l'appropriation de nouveaux sons et que l'âge n'est pas un critère déterminant.

Dans la section suivante, nous présentons les systèmes des sons de deux langues, la langue française et la langue serbe, afin de désigner le contraste qui existe entre ces deux systèmes des voyelles.

\section{SYSTEMES VOCALIQUES FRANÇAIS ET SERBE}

«Le français se caractérise par l'antériorité, la labialité, la nasalité et la tension musculaire » (Guimbretière 1996, 23). Le système des sons de la langue française est composé de 36 phonèmes, dont 16 voyelles, 3 semi-voyelles et 17 consonnes. Le système vocalique est fait de 16 voyelles dont 12 voyelles orales et 4 nasales. Selon les caractéristiques articulatoires, on peut diviser toutes les voyelles de la manière suivante : très fermées $[i, u, y]$, fermées [e, $\varnothing, o, \tilde{o}]$, moyennes [ə], ouvertes $[\varepsilon, \propto, \rho, \tilde{\varepsilon}$, œ̃] et très ouvertes [a, a, $\tilde{\alpha}$ ] (Léon M. et Léon P. 2009, 22). Comme on peut voir, certaines voyelles orales se distinguent par l'aperture, la voyelle /E/ connait ses réalisations fermées et ouvertes $[\mathrm{e}, \varepsilon]$, tout comme la voyelle $/ \mathrm{O} /[\mathrm{o}$, ๖] et la voyelle /E/ [ø, œ]. La voyelle [ə] caduc ou instable est une voyelle prononcée dans certaines positions du mot. La voyelle [a] antérieur a remplacé la prononciation de [a] postérieur, sauf dans les cas où il s'agit des oppositions phonologiques distinctives, si l'on se réfère à la prononciation du français standard. Le [a] postérieur est prononcé dans les parlers régionaux et en français québécois (Gudurić 2009, 93-100). On peut décrire l'articulation des voyelles nasales de la manière

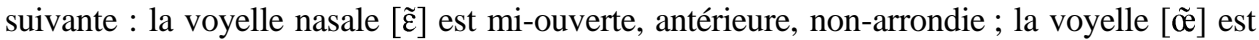
mi-ouverte, antérieure, arrondie; la voyelle $[\tilde{\alpha}]$ est ouverte, postérieure, non-arrondie ; la voyelle [̃̃] est mi-ouverte, postérieure, arrondie. Les quatre voyelles nasales, ainsi que le [a] postérieur, le [o] fermé et le [ø] fermé, possèdent la durée naturelle, c'est-à-dire que ces voyelles sont longues dans la syllabe fermée accentuée, devant n'importe quelle consonne prononcée, tandis que les autres voyelles possèdent la durée combinatoire: elles sont allongées devant les consonnes [R, $\mathrm{z}, \mathrm{v}, \mathrm{3}]$ ou devant le groupe consonantique [VR] ou [vl]. Il faut noter que cela ne concerne pas le [e] fermé et le [ə] instable, car on ne les trouve jamais dans une syllabe fermée accentuée (Gudurić 2009).

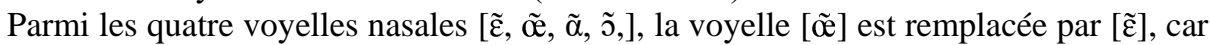
le nombre de mots dans lesquels ce phonème possède une valeur phonologique et distinctive est très restreint (Léon 1966, 39 ; Gudurić 2009, 118-119). Dans les médias

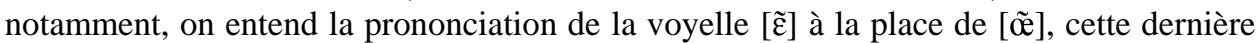
étant complètement remplacée (Detey et al. 2010a, 151). Il faut noter aussi la fréquence 
de toutes les quatre voyelles nasales, ce qui peut expliquer aussi la substitution de [õ] par $[\tilde{\varepsilon}]$, car c'est une des nasales avec la plus faible fréquence : $[\tilde{\alpha}]-3,3 \%,[\tilde{\jmath}]-2 \%,[\tilde{\varepsilon}]-$ $1,4 \%,[\tilde{\varrho}]-0,5 \%$ (Catach [1980] 2016, 107).

Les auteurs des manuels de phonétique et phonologie du français proposent les exercices d'articulation et discrimination des trois voyelles nasales $[\tilde{\varepsilon}, \tilde{\alpha}, \tilde{\jmath}]^{1}$, et parmi les graphies se rapportant aux sons on ne trouve pas les graphies correspondantes à la voyelle

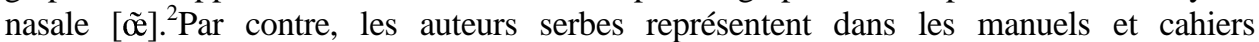

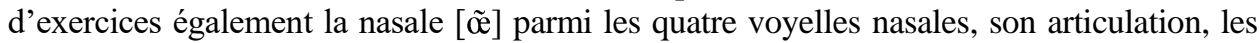
activités de discrimination ainsi que les graphies correspondantes, même si elle a été remplacée par $[\tilde{\varepsilon}]{ }^{3}$ Pour atteindre une bonne prononciation des segments, Šotra a développé les stratégies d'acquisition, en signalant qu'il est important que les apprenants serbes

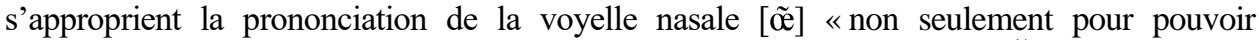
l'articuler précisément mais aussi pour développer sa perception auditive » (Šotra 2006, 102).

Le système des sons de la langue serbe est composé de 30 phonèmes, dont cinq voyelles orales [a, e, i, o, u]. Selon l'aperture, on distingue les voyelles fermées [i, u], les voyelles moyennes [e, o] et la voyelle ouverte [a], tandis que selon le point d'articulation on les classifie dans trois catégories : antérieures [i, e], postérieures [u, o] et moyenne [a]. Les voyelles postérieures sont labialisées, tandis que les lèvres sont neutres si l'on prononce la voyelle antérieure [e], peu écartées pour la voyelle [i] et finalement ouvertes, si l'on prononce la voyelle moyenne [a] (Subotić et al. 2012, 44-45). D'après Miletić, le système des voyelles serbes, qui ne possède que cinq voyelles, représente un obstacle pour apprendre les langues étrangères, notamment le français car, au niveau de la perception auditive, les locuteurs dont le serbe est la langue maternelle ont des difficultés à distinguer les voyelles à double timbre (Miletić 1960, 20).

Donc, dans le système vocalique de la langue serbe on ne trouve pas de voyelles nasales, ce qui en fait un contraste évident par rapport au système des voyelles du français. Les voyelles nasales du français sont caractérisées par la présence de la résonance nasale, tandis qu'en serbe et dans d'autres langues la résonance nasale est le résultat de la proximité des consonnes nasales [m] ou [n], ou bien elle est la caractéristique individuelle sans valeur linguistique ou sémantique (Malmberg 1954, 42-44). Cela veut dire que les voyelles nasales du français sont des phonèmes du système vocalique, tandis qu'en serbe il s'agit des variantes combinatoires liées à la coarticulation, dont témoigne la prononciation du prénom serbe Stanko où l'on a la réalisation de la voyelle nasalisée en contact avec la consonne nasale (Gudurić 2004, 82-85). Tout compte fait, la perception et la production des voyelles nasales du français peuvent représenter un maillon faible dans l'acquisition de ces voyelles par les apprenants serbes, faute de phonèmes équivalents en serbe. Pourtant, le fait que le serbe possède les réalisations nasalisées en contexte pourrait être un facteur facilitant dans l'appropriation de ce paramètre.

Dans le chapitre suivant, nous présentons les recherches précédentes sur les voyelles nasales du français.

\footnotetext{
${ }^{1}$ Voir dans Abry, Veldeman-Abry 2007 ; Abry, Chalaron 2010 ; Abry, Chalaron 2011

${ }^{2}$ Voir dans Abry, Veldeman-Abry 2007 : 41-42.

${ }^{3}$ Voir dans Šotra 2006 ; Gudurić, Radusin 2008 ; Gudurić 2009 ; Ignjatović 2018.
} 


\section{RECHERCHES PRECEDENTES SUR LA PERCEPTION ET \\ LA PRODUCTION DES VOYELLES NASALES DU FRANÇAIS}

Étant donné que les voyelles nasales représentent plutôt une exception qu'une règle propre aux systèmes vocaliques des langues, les apprenants qui étudient le français langue étrangère éprouvent les difficultés, d'où provient le besoin des chercheurs de s'occuper de cette problématique. Depuis ces dernières années, les recherches qui concernent la perception et la production des voyelles nasales par les apprenants de français langue étrangère ainsi que les analyses acoustiques, ont été faites dans les contextes variés, auprès des locuteurs de différentes langues maternelles apprenant le français langue étrangère, par exemple : Detey et al. (2010b), Brkan et al. (2012), Bustamante et al. (2014), Bustamante et al. (2018), Petersen (2015), Kakoyianni-Doa et al. (2017), Nawafleh, Alrabadi (2017) ${ }^{4}$.

Dans une étude menée sur la perception des voyelles nasales du français par des locuteurs espagnols et colombiens, Bustamante et al. concluent que ces sujets perçoivent

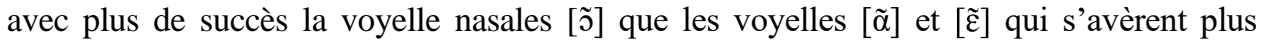
problématiques (Bustamante et al. 2018)

L'article de Kakoyianni-Doa et al. démontre les difficultés éprouvées par les étudiants

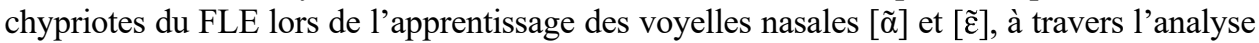
des deux systèmes vocaliques, du français et de leur langue maternelle, l'analyse de la perception ainsi que les propositions de surmonter ces difficultés. Suivant ces résultats, les voyelles nasales $[\tilde{\alpha}]$ et $[\tilde{\varepsilon}]$ ont été correctement discriminées dans deux tiers de mots du corpus, les étudiants ont distingué la voyelle orale de la voyelle nasale [ã ] dans $91 \%$ d'occurrences et la voyelle orale de la voyelle nasale [ $\tilde{\varepsilon}]$ dans $70-84 \%$ d'occurrences. Les auteurs considèrent qu'il est important de relier les sons avec les graphies correspondantes afin de mieux acquérir les voyelles nasales (Kakoyianni-Doa et al. 2017).

Les locutrices du bosniaque, langue maternelle, distinguent les trois voyelles nasales, ayant une prononciation très proche des natifs, ce qui est dû, d'après les auteures, à leurs compétences linguistiques avancées, du niveau B2 et $\mathrm{C} 1$. Quant à la durée des voyelles, elles constatent que les locutrices enquêtées tendent à allonger d'avantage les voyelles nasales que les locutrices natives (Brkan et al. 2012).

Une étude acoustique et perceptive des trois voyelles nasales, dont l'objectif primaire était d'analyser la perception et production des locuteurs hispanophones et japanophones, a

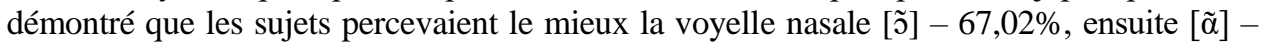
$54,53 \%$ et à la fin $[\tilde{\varepsilon}]-51,27 \%$ (Detey et al. 2010b). En se référant à Hansen (2001) et sa recherche sur la production des voyelles nasales par les locuteurs natifs, Detey et al. constatent :

«Il n'est en outre pas exclu que le changement phonétique en chaîne subi par les voyelles nasales en français standard contemporain (Hansen, 2001) favorise les

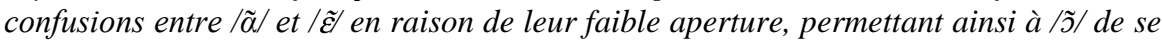
distinguer perceptivement par un arrondissement et une fermeture plus marqués. La catégorie /̃̃/ semblerait donc être globalement la moins problématique et la mieux identifiée » (Detey et al. 2010b, 1297).

\footnotetext{
${ }^{4}$ Il faut noter ici que dans l'article de Nawafleh, Alrabadi (2017), il s'agit d'une étude sur de la perception et production de toutes les voyelles du français, orales et nasales.
} 
Fónagy voulait vérifier si ces voyelles se rapprochaient, si les locuteurs parisiens remplaçaient une voyelle nasale par l'autre. D'après les résultats de deux tests on peut

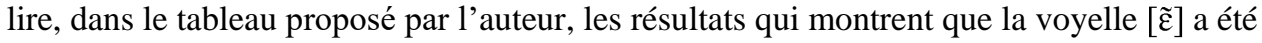
le mieux perçue au total, ensuite [ã] et à la fin [̃̃] (Fónagy 1989, 230).

Dans les chapitres suivants nous allons présenter les objectifs de notre recherche, les sujets, le corpus et la méthodologie.

\section{PRESENTE RECHERCHE}

L'objectif de la présente recherche est d'analyser la perception des voyelles nasales par les étudiants serbes de français langue étrangère. Autrement dit, la perception précédant la production constitue un paramètre nécessaire à l'acquisition des phonèmes.

\subsection{Participants}

Nous avons fait cette recherche au Département de langue et littérature françaises, à la Faculté de Philosophie de l'Université de Niš. Plus précisément, vingt étudiants en première année, pendant deux années académiques consécutives, 2017/2018 et 2018/2019, ont participé à cette expérience. Cette recherche avait deux objectifs : 1) comparer la perception des voyelles nasales par deux groupes d'étudiants ; 2) comparer les résultats de deux groupes par rapport à la position des voyelles nasales dans les mots. Les étudiants en première année académique durant l'année 2017/2018 seront désignés comme groupe 1 et les étudiants en première année durant l'année académique 2018/2019 seront désignés comme groupe 2. Tous les participants de l'enquête apprenaient le français langue étrangère depuis la classe de cinquième, niveau collège, en tant que deuxième langue étrangère, c'està-dire depuis huit ans avant l'inscription aux études de langue et littérature françaises.

\subsection{Corpus}

Le corpus a été composé de deux tests de perception :

a) Le premier test de perception a été fait de 10 paires de mots par voyelle, se différenciant par une seule voyelle orale/nasale à la position interconsonantique et en finale absolue, soit au total 30 paires de mots. On a demandé aux sujets d'entourer la bonne réponse en fonction du mot entendu, ayant la voyelle orale $[\varepsilon, a, o / s$,$] ou nasale [\tilde{\varepsilon}, \tilde{\alpha}, \tilde{o}]$, selon le cas. Nous n'avons pas proposé les mots ayant la voyelle nasale [ã], pour des raisons argumentées plus haut : le nombre de mots où ce phonème a un rôle distinctif est restreint et de plus, il est le plus souvent remplacé par le phonème $[\tilde{\varepsilon}]$.

b) Le deuxième test de perception a été fait de trois activités dont chacune a été composée

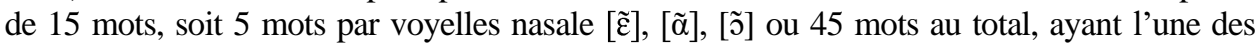
trois voyelles nasales en trois positions du mot (initiale, interconsonantique et finale absolue).

\subsection{Méthodologie et procédure}

La présente recherche est basée sur la perception des sons, la discrimination des voyelles orales/nasales, l'identification des voyelles nasales. On a procédé de la manière suivante : nous avons lu les listes de mots en proposant aux participants d'entourer la bonne réponse en fonction de la voyelle entendue. Pour le premier test, ils avaient un choix binaire, entre deux mots écrits, où l'un des deux mots dans la paire minimale avait une voyelle orale ou nasale à 
la position interconsonantique ou en finale absolue, par exemple : cette/sainte, mais/main; chasse/chance, chat/chant; mode/monde, peau/pont. Pour faire le deuxième test, on a proposé trois activités pour les trois positions du mot, initiale, interconsonantique et finale absolue. On a lu 15 mots par activité, 5 mots par voyelle, et la tâche consistait à cocher les cases selon la voyelle nasale entendue, les symboles API des voyelles nasales étant inscrits dans les colonnes respectives. Voici quelques exemples de mots proposés ayant l'une des voyelles nasales à l'initiale: instable, ombrage, ambitieux, à la position interconsonantique : linge, banque, ronde, en finale absolue : main, vent, long. Dans les sections qui suivent, nous allons présenter les résultats et les interprétations de la recherche.

\subsection{Résultats de la recherche}

Le premier test de perception consistait en identification des voyelles orales/nasales en deux positions du mot : à l'interconsonantique et en finale absolue. Les résultats montrent que les étudiants du groupe 1 perçoivent la différence entre la voyelle orale [a] et la voyelle nasale $[\tilde{\alpha}]$ dans les deux positions du mot, la réussite étant $100 \%$.

Les voyelles $[\varepsilon]$ et $[\tilde{\varepsilon}]$, ont été correctement repérées à la position interconsonantique, tandis qu'en finale absolue ces voyelles ont été clairement discriminées dans $90 \%$ d'occurrences. Plus précisément, on a noté une fausse réponse par paire, dans les oppositions de mots : mais/main, paix/pain et baie/bain.

Le premier groupe d'étudiants a éprouvé le plus de difficultés à discriminer la voyelle

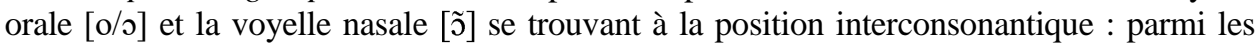
cinq paires de mots proposés, on a noté huit bonnes réponses : autrement dit, dans $80 \%$ d'occurrences les sujets ont correctement identifié la voyelle orale/nasale. À la position finale, on a noté $9 / 10$ bonnes réponses, à part l'opposition beau/bon où sept étudiants ont correctement identifié la voyelle nasale du mot lu, tandis que trois étudiants ont entouré la fausse réponse, le mot beau.

En ce qui concerne le deuxième groupe d'étudiants, les résultats du premier test de perception montrent que tous les étudiants différencient les trois voyelles nasales des voyelles orales à la position interconsonantique et en finale absolue, la réussite s'élève à $100 \%$, ou autrement dit, toutes les voyelles ont été correctement repérées.

Le deuxième test de perception a été composé de mots ayant les trois voyelles nasales en trois positions : à l'initiale, à l'interconsonantique et en finale absolue du mot.

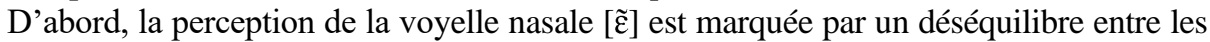
deux groupes en position interconsonantique : le premier groupe ayant perçu ce phonème dans $40 \%$ de cas contre le deuxième groupe dont le pourcentage s'élève à $74 \%$; soit au total, la valeur moyenne dans cette position fait $57 \%$ d'occurrences, pour les deux groupes. En position initiale et en finale absolue les deux groupes ont éprouvé quasiment le même taux de réussite: soit au total, pour les deux groupes, les valeurs moyennes font $71 \%$ d'occurrences à l'initiale et $52 \%$ en finale absolue. Au total, y compris les trois positions, cette voyelle a été correctement perçue dans 52,6\% d'occurrences (groupe 1) et 67,3\% (groupe 2), soit presque $60 \%$ de réussite pour les deux groupes, la valeur moyenne, en trois

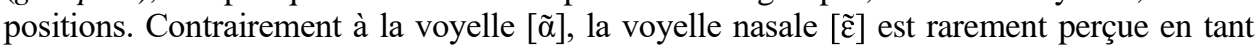
que la voyelle nasale [ว]], en trois positions les résultats de deux groupes d'étudiants le démontrent. Si elle est remplacée par la voyelle [ã], cela est le plus évident à la position finale absolue dans presque la moitié d'occurrences, précisément dans $45 \%$ pour les deux groupes, ensuite en position interconsonantique la valeur moyenne pour les deux groupes 
est $40 \%$, et finalement à l'initiale c'est $24 \%$ pour les deux groupes. Au total, pour les deux groupes et en trois positions, la voyelle $[\tilde{\varepsilon}]$ a été perçue en tant que la voyelle nasale $[\tilde{\alpha}]$ dans $36,3 \%$ d'occurrences. Ces résultats nous permettent de constater que la discrimination de ce phonème s'avère la plus problématique pour les deux groupes d'étudiants.

Tableau 1 : Perception de la voyelle nasale $[\tilde{\varepsilon}]$

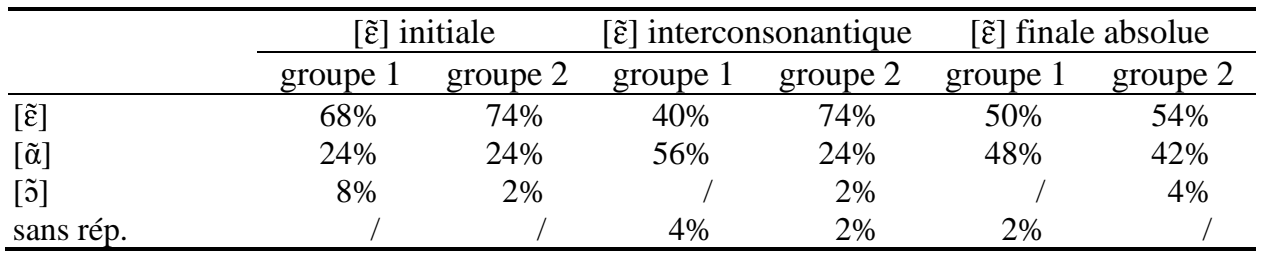

Quant à la voyelle nasale [ã], on remarque plutôt un équilibre dans sa perception à la position initiale, par les deux groupes de sujets : elle a été correctement identifiée dans $62 \%$ d'occurrences par le groupe 1 et $60 \%$ par le groupe 2. En position interconsonantique, le groupe 2 a eu plus de succès que le groupe $1: 90 \%$ d'occurrences contre $78 \%$, tandis qu'en position finale absolue du mot le groupe 1 a correctement identifié la voyelle nasale [ã] dans $64 \%$ d'occurrences mais pour le groupe 2 on a noté $80 \%$ d'occurrences. Au total, la valeur moyenne de perception de cette voyelle, y compris les trois positions, s'élève à $68 \%$ d'occurrences (groupe 1) et 76,6\% d'occurrences (groupe 2), soit 72,3\%, valeur moyenne pour les deux groupes. C'est-à-dire que les deux groupes ont quasiment le même taux de réussite dans la discrimination de ce phonème. Quant à la discrimination erronée, si l'on compare les résultats de deux groupes, on peut voir que la voyelle nasale $[\tilde{\alpha}]$ est remplacée par

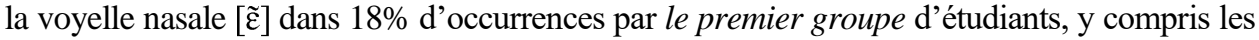
trois positions, tandis que la valeur moyenne pour le deuxième groupe d'étudiants fait 6,6\%, en notant qu'il n'y avait pas d'erreurs à la position interconsonantique. Les valeurs moyennes où cette voyelle est remplacée par la voyelle nasale [õ] font 14\% (groupe l) et 15,3\% (groupe 2 ), donc il s'agit des résultats très approximatifs. Si l'on compare les résultats entre ces deux groupes selon les erreurs commises et la position du mot, on voit que le premier groupe a eu

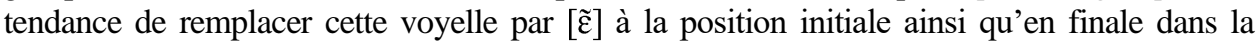
plupart de cas, tandis que le deuxième groupe a montré le plus grand nombre d'erreurs à

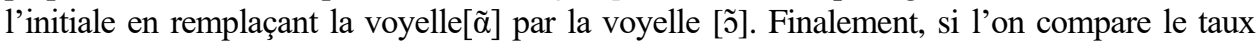
d'erreurs éprouvées par les deux groupes au total, par position du mot, on voit bien que les

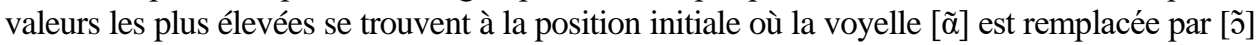

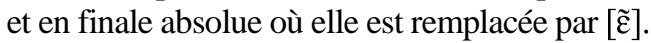

Tableau 2 : Perception de la voyelle nasale $[\tilde{\alpha}]$

\begin{tabular}{lcrrrrr}
\hline & \multicolumn{2}{c}{$[\tilde{\alpha}]$ initiale } & \multicolumn{2}{c}{$[\tilde{\alpha}]$ interconsonantique } & \multicolumn{2}{c}{$[\tilde{\alpha}]$ finale absolue } \\
\cline { 2 - 7 } & groupe 1 & groupe 2 & groupe 1 & groupe 2 & groupe 1 & groupe 2 \\
\hline$[\tilde{\alpha}]$ & $62 \%$ & $60 \%$ & $78 \%$ & $90 \%$ & $64 \%$ & $80 \%$ \\
{$[\tilde{\varepsilon}]$} & $24 \%$ & $8 \%$ & $8 \%$ & $/$ & $22 \%$ & $12 \%$ \\
{$[\tilde{\jmath}]$} & $14 \%$ & $30 \%$ & $14 \%$ & $10 \%$ & $14 \%$ & $6 \%$ \\
sans rép. & $/$ & $2 \%$ & $/$ & $/$ & $/$ & $2 \%$ \\
\hline
\end{tabular}


En comparant les résultats de perception de deux voyelles précédentes avec ceux de la voyelle nasale [̃]], on voit bien que cette dernière a été perçue le plus correctement en trois positions, les valeurs moyennes étant de $90 \%$ à $97 \%$ d'occurrences par position. On remarque aussi un déséquilibre dans l'identification de ce phonème à la position interconsonantique entre les deux groupes : 80\% d'occurrences (groupe 1) et 100\% (groupe 2). On note donc un léger avantage au profit du deuxième groupe, car en trois positions la réussite s'élève à $98 \%$, tandis que pour le groupe 1 c'est $89,3 \%$. On note aussi que le premier groupe a eu tendance de remplacer cette nasale par la nasale $[\tilde{\alpha}]$ dans $12 \%$ de cas, ce qui représente la valeur moyenne pour les trois positions, tandis que ce n'était pas le cas avec le deuxième groupe. Donc, cette voyelle a été la mieux perçue par les deux groupes d'étudiants en trois positions du mot.

Tableau 3 : Perception de la voyelle nasale [õ]

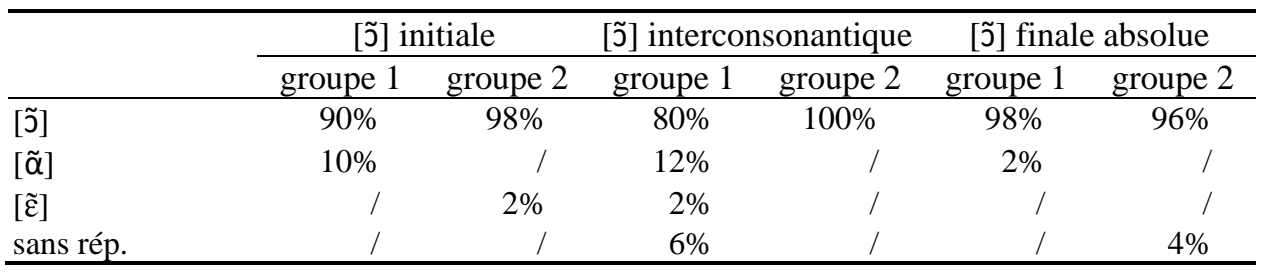

Si l'on regarde les résultats de perception des trois voyelles nasales en trois positions, par deux groupes d'étudiants, on voit bien que la voyelle [õ] vient en tête avec 93,6\% de réussite, ensuite c'est la voyelle $[\tilde{\alpha}]$ avec $72,3 \%$ et à la fin la voyelle nasale $[\tilde{\varepsilon}]$ avec $60 \%$ de réussite.

\section{DISCUSSION}

Selon les résultats de perception des trois voyelles nasales $[\tilde{\varepsilon}, \tilde{\alpha}, \tilde{o}]$ par les deux groupes d'étudiants qu'on vient d'exposer, on peut voir qu'ils perçoivent et distinguent bien les voyelles orales des voyelles nasales. On en voit deux raisons possibles : d'abord, le trait distinctif oralité/nasalité des voyelles représente un paramètre que les étudiants serbes ont remarqué avec facilité, ce qui est dû au fait que les réalisations nasalisées existent dans leur langue maternelle. Pourtant, il ne s'agit pas de sons identiques mais similaires qui pouvaient être classifiés dans les prototypes existants dans leur langue maternelle. Ensuite, il s'agit des étudiants qui ont déjà intégré ce trait dans leur système perceptif pendant l'apprentissage du français. De plus, pour effectuer cette tâche, les étudiants avaient un test binaire, c'est à dire qu'on leur avait proposé deux mots orthographiés et ils devaient choisir la bonne réponse en fonction du mot entendu, donc ils ont dû relier le mot entendu avec sa représentation graphique, relier la perception auditive avec les graphies, ce qui a contribué à leur réussite.

De l'autre côté, les difficultés s'avèrent importantes si les étudiants doivent identifier la voyelle entendue, parmi les trois voyelles proposées. Ici, ils devaient mettre une croix dans la case où figuraient les symboles API des trois voyelles. Donc, cette tâche n'était pas facilitée par les mots orthographiés, car on sait d'avantage que les apprenants, en général, sont plutôt focalisés sur l'écrit que sur l'oral, et nous avons voulu vérifier la discrimination des voyelles nasales entre elles, sans support écrit. 
Les résultats montrent qu'ils discriminent mieux les voyelles nasales postérieures, [õ] et $[\tilde{\alpha}]$ que la nasale antérieure $[\tilde{\varepsilon}]$. La voyelle nasale la plus confondue avec les deux autres c'est la voyelle $[\tilde{\alpha}]$, tandis que la voyelle la moins correctement discriminée et remplacée notamment par la voyelle $[\tilde{\alpha}]$, c'est la voyelle $[\tilde{\varepsilon}]$.

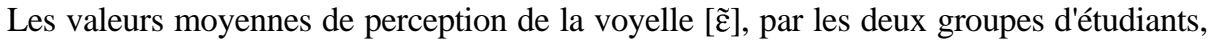
en trois positions du mot (initiale, interconsonantique et finale), montrent que cette nasale a été correctement identifiée dans $60 \%$ d'occurrences, tandis qu'elle a été remplacée par [ã ] dans $36,3 \%$ de cas et par [0̃] dans $2,6 \%$ de cas, au total. Selon la position du mot, on voit que cette nasale est remplacée par [ã], surtout en position interconsonantique, la valeur moyenne pour les deux groupes fait $40 \%$, et en position finale absolue la valeur moyenne pour les deux groupes est $45 \%$.

Les valeurs moyennes de perception de la voyelle $[\tilde{\alpha}]$ par les deux groupes d'étudiants, en trois positions du mot, montrent que cette nasale a été correctement perçue dans $72,3 \%$

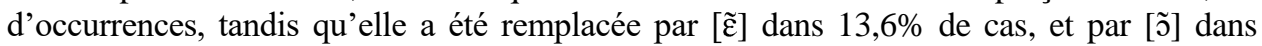
$14,6 \%$ de cas, au total.

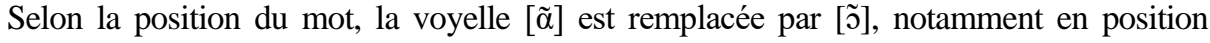
initiale, la valeur moyenne fait $22 \%$ pour les deux groupes, et en finale absolue où elle est remplacée par [ $\tilde{\varepsilon}]$, la valeur moyenne pour les deux groupes est $17 \%$. On peut expliquer ces résultats comme suit: la voyelle [a]] se différencie par rapport à la voyelle orale serbe /a/ d'abord par nasalité, ensuite cette dernière est centralisée tandis que la voyelle $[\tilde{\alpha}]$ est postérieure. Ceci dit que ces apprenants avaient du mal à acquérir le paramètre de postériorité de cette voyelle et alors, ils l'ont remplacée par la nasale [õ] à l'initiale, car en comparant avec le serbe, la postériorité est une caractéristique propre à la voyelle /o/ en serbe. Il ne s'agit pas d'un pourcentage insignifiant, notamment pour le deuxième groupe $(30 \%)$.

Cette confusion entre $[\tilde{\varepsilon}]$ et $[\tilde{\alpha}]$, notamment dans les cas où la voyelle $[\tilde{\varepsilon}]$ est remplacée par

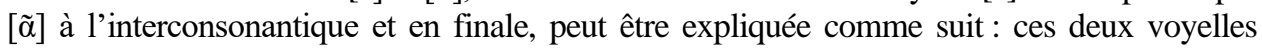
nasales sont caractérisées par les traits communs : la nasalité et la non labialisation, et par les traits distinctifs : voyelle ouverte/mi-ouverte, postérieure/antérieure. Pour prononcer la nasale [ש̃] les lèvres sont écartées, tandis que pour le /e/ serbe les lèvres restent neutres, ce qui nous permet de constater qu'il s'agit d'un paramètre non acquis par les apprenants serbes, car l'écartement n'est pas un trait propre à la voyelle /e/ de la langue serbe. De plus, on a étudié les mots où les étudiants ont confondu les voyelles $[\tilde{\varepsilon}]$ et $[\tilde{\alpha}]$ dans la plupart de cas. Les mots où la voyelle $[\tilde{\varepsilon}]$ est remplacée par $[\tilde{\alpha}]$ en finale absolue, la position dans laquelle on note le plus grand pourcentage d'erreurs, sont main et pain, à l'interconsonantique mince et linge. La nasale $[\tilde{\alpha}]$ est remplacée par $[\tilde{\varepsilon}]$ en position finale dans le plus grand nombre d'occurrences et il s'agit des mots vent et banc. Ensuite, elle est remplacée par [õ] à l'initiale dans le plus grand nombre d'occurrences, il s'agit des mots enceinte, ambitieux et ancêtre.

Les valeurs moyennes de perception de la voyelle [õ] par les deux groupes d'étudiants, en trois positions du mot, montrent que la voyelle nasale [0̃] a été correctement perçue dans

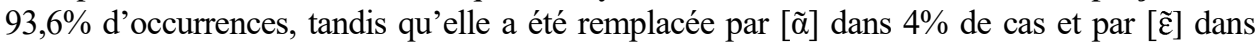
$0,6 \%$ de cas, au total. La réussite très élevée dans l'identification de la nasale [̃̃] peut être expliquée par le fait que ce phonème a été assimilé dans la catégorie similaire de la langue maternelle des étudiants : les caractéristiques communes que la voyelle nasale [õ] possède avec la voyelle orale /o/ du serbe sont l'aperture moyenne, la postériorité et l'arrondissement, le seul trait de dissemblance c'est la nasalité. 


\section{CONCLUSION}

Cette recherche montre que même si dans la langue maternelle des apprenants les voyelles nasales n'existent pas, ils sont capables de discriminer ces phonèmes de la langue cible en faisant une nette différence entre les voyelles orales et nasales : d'une part, c'est grâce au contraste par rapport à la langue serbe qui ne connait pas les voyelles nasales comme phonèmes distinctifs, et d'autre part, cela est dû au fait que les locuteurs du serbe langue maternelle possèdent les réalisations nasalisées dans leur système perceptif.

D'abord, nous avons mis en relation l'identification de trois voyelles nasales aves le système vocalique de la langue serbe et nous avons démontré que les étudiants avaient assimilé ces voyelles dans les catégories existantes dans leur langue maternelle, en les intégrant le plus souvent par traits communs. De plus, l'étude de la perception des trois voyelles nasales se trouvant à la position initiale, à l'interconsonantique et en finale absolue des mots proposés, a révélé des résultats très intéressants, que nous pouvons considérer universels, en comparant avec les autres recherches menées, dont les résultats nous avons évoqués dans cet article.

Donc, d'après les résultats de notre recherche, la voyelle nasale perçue avec le plus grand

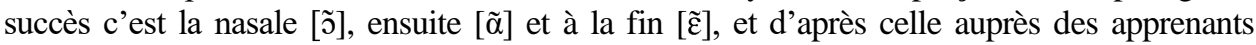
hispanophones et japanophones (Detey et al. 2010b), l'ordre des voyelles nasales les mieux perçues demeure le même. Ensuite, encore une étude auprès des espagnols et colombiens

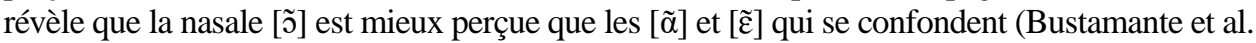
2018). La langue espagnole possède cinq voyelles orales et ne possède pas de voyelles nasales, tout comme la langue serbe, alors, il faudrait peut-être chercher une explication parmi les similarités entre les systèmes vocaliques serbe et espagnol afin de justifier les résultats obtenues dans l'identification des voyelles nasales des apprenants serbophones et hispanophones. En outre, on a vu dans une étude sur la production de la parole chez les natifs qu'ils éprouvaient eux-mêmes la tendance de confondre $[\tilde{\alpha}]$ et $[\tilde{\varepsilon}]$ « en raison d'une faible aperture » et que la voyelle [̃̃] est mieux perçue, étant plus arrondie que les deux autres (Hansen 2001 dans Detey et al. 2010b).

Finalement, même si le changement des voyelles $[\tilde{\alpha}]$ et $[\tilde{\varepsilon}]$ n'est pas une caractéristique

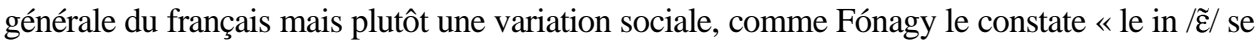
déplace souvent vers an / $\tilde{\alpha} /$, surtout dans la parole de jeunes Parisiens » (Fónagy 1989, 226), on peut se demander s'il faudrait insister sur la correction, sauf s'il s'agit de paires minimales où le remplacement d'un phonème par l'autre produirait des ambiguïtés ou même perturberait le sens (par ex. vin/vent).

Pour les prochaines recherches, il faudra prendre en compte les expériences sur la production des voyelles nasales, afin de comparer la relation entre la perception et la production, l'influence de la perception sur la production, vérifier aussi si les étudiants

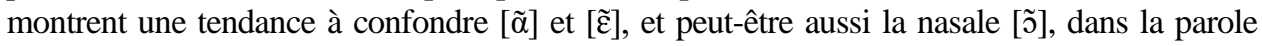
spontanée.

Note : Cet article est rédigé dans le cadre du projet scientifique Les langues, les littératures et les cultures romanes et slaves en contact et en divergence, $N^{o}$ 81/1-17-8-01, approuvé par la Faculté de Philosophie de l'Université de Niš et soutenu par l'Agence universitaire de la Francophonie. 


\section{BIBLIOGRAPHIE}

Abry, Dominique et Marie-Laure Chalaron. Les 500 exercices de phonétique A1/A2. Paris, Hachette 2010.

Abry, Dominique et Marie-Laure Chalaron. D. Les 500 exercices de phonétique B1/B2. Paris, Hachette, 2011.

Abry, Dominique et Julie Veldeman-Abry. La phonétique : audition, prononciation, correction. Paris, Clé International, 2007.

Best, Catherine. «The emergence of native-language phonological influences in infants: A perceptual assimilation model ». Haskins Laboratories Status Report on Speech Research 1991, SR 107/ 108, 1-30, 1994. Disponible sur : https://www.researchgate.net/publication/243772232

Brkan, Altijana, Angélique Amelot et Claire Pillot-Loiseau . «Utilisation d'un accéléromètre piézoélectrique pour l'étude de la nasalité du Français Langue étrangère », dans Conférence conjointe JEP-TALN-RECITAL 2012, volume 1, JEP, Grenoble, 4 au 8 juin 2012, p. 689-696. Disponible sur : https://www.aclweb.org/anthology/F121087

Bustamante, David Alejandro, Angélique Amelot et Claire Pillot-Loiseau. «Étude de la production des voyelles nasales du français chez des apprenantes espagnoles et colombiennes », dans $X X X^{e}$ édition des Journées d'Etudes sur la Parole, Le Mans, 23-27 juin 2014, Laboratoire d'Informatique de l'Université du Maine (LIUM) et le Laboratoire d'Informatique de Nantes Atlantique (LINA) sous l'égide de l'Association Francophone de la Communication Parlée (AFCP), Jun 2014, Le Mans, France. p.576/80. Disponible sur: https://hal.archivesouvertes.fr/hal-01136740

Bustamante, David Alejandro, Pierre Hallé et Claire Pillot-Loiseau. « Perception des voyelles nasales du français par des apprenants hispanophones », dans Conférence, XXXII ' Journées d'Études sur la Parole, 2018. Disponible sur :

https://www.researchgate.net/publication/325552070_Perception_des_voyelles_nasales_du_francais_par_des_apprena nts_hispanophones

Catach, Nina. L'orthographe française. Traité théorique et pratique. Paris, Armand Colin, [1980] 2016.

Cornaire, Claudette. La compréhension orale. Paris, Clé International, 1998.

Detey, Sylvain, Jacques Durand, Bertrand Laks et Chantal Lyche. Les variétés du français parlé dans l'espace francophone, ressources pour l'enseignement. Paris, Ophrys, 2010a.

Detey, Sylvain, Isabelle Racine, Yuji Kawaguchi, Françoise Zay et Nathalie Buehler. «Évaluation des voyelles nasales en français L2 en production : de la nécessité d'un corpus multitâches », dans Neveu F., Muni Toke V., Durand J., Klingler T., Mondada L., Prévost S., Congrès Mondial de Linguistique Française. Institut de Linguistique Française, Phonétique, phonologie et interfaces. Paris, 2010b, p. 1289-1301. Disponible sur :

https://www.researchgate.net/publication/264936445_Evaluation_des_voyelles_nasales_en_francais_L2_en_productio n_de_la_necessite_d'un_corpus_multitaches

Flege, James Emil. « Assessing constraints on second-language segmental production and perception », dans Phonetics and Phonology in Language Comprehension and Production, éd. par Niels O. Schiller et Antje S. Meyer, 2003, p. 319-355. Berlin, New York, Mouton de Gruyter.

Fónagy, Ivan. Le français change de visage. Revue Romane 24, 2, 1989. Disponible sur : https://tidsskrift.dk/revue romane/article/view/29677/26966

Gudurić, Snežana. O prirodi glasova. Beograd, Zavod za udžbenike i nastavna sredstva, 2004

Gudurić, Snežana. Osnovi fonetike s fonologijom francuskog jezika. Beograd, Zavod za udžbenike i nastavna sredstva, 2009.

Gudurić, Snežana et Nataša Radusin. Phonétique et phonologie de la langue française, cahier d'exercices. Novi Sad, Filozofski fakultet, 2008.

Guimbretière, Élisabeth. Phonétique et enseignement de l'oral. Paris, Didier/Hatier, 1996.

Hansen, Anita Berit. Les changements actuels des voyelles nasales du français parisien : confusions ou changement en chaîne ? La linguistique 2001/2 (Vol. 37, p. 33-48. Disponible sur : https://www.cairn.info/revue-la-linguistique2001-2-page-33.htm

Ignjatović, Nataša. Phonétique et orthographe 1 pour les étudiants de FLE, cahier d'exercices. Niš, Filozofski fakultet, 2018

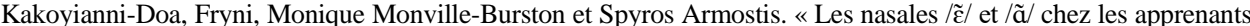
hellénophones ». Revue du Centre Européen d'Etudes Slaves- La revue Numéro 6. [En ligne] Publié en ligne le 06 mars 2017. Disponible sur : http://etudesslaves.edel.univ-poitiers.fr/index.php?id=1108

Kuhl, Patricia. A new view of language acquisition.PNAS October 24, 200097 (22) 11850-11857 ; Disponible sur : https://doi.org/10.1073/pnas.97.22.11850

Léon, Monique et Pierre Léon. La prononciation du français. Paris, Armand Colin, 2009.

Léon, Pierre. Prononciation du français standard. Paris, Librairie Marcel Didier, 1966.

Lauret, Bertrand. Enseigner la prononciation du français : questions et outils. Paris, Hachette, 2007.

Malmberg, Bertil. La phonétique. Paris, Presses Universitaires de France, 1954.

Miletić, Branko. Osnovi fonetike srpskog jezika. Beograd, Naučna knjiga, 1960. 
Nawafleh, Ahmad et Elie Alrabadi. Étude acoustique et perceptive des voyelles du français réalisées par des apprenants jordaniens et des locuteurs français. Çedille. Revista de Estudios Franceses, 2017. Disponible sur : http://www.redalyc.org/html/808/80850903018/

Petersen, Stacy. Categorical Nasal Vowel Acquisition in L2 French Learners, article 25, University of Pennsylvania Working Papers in Linguistics, Volume 21, Issue 1, Penn Libraries, 2015. Disponible sur : https://repository.upenn.edu/cgi/viewcontent.cgi?article=1854\&context=pwpl

Subotić, Ljiljana, Dejan Sredojević i Isidora Bjelaković. Fonetika i fonologija: ortografska i ortoepska norma standardnog srpskog jezika. Novi Sad, Filozofski fakultet, 2012.

Trubetzkoy, S. Nicolas. Principes de phonologie. Paris, Klincksieck, [1938] 2005.

Šotra, Tatjana. Kako progovoriti na stranom jeziku. Beograd, Zavod za udžbenike i nastavna sredstva, 2006.

\section{PERCEPCIJA FRANCUSKIH NAZALNIH VOKALA KOD SRPSKIH STUDENATA FRANCUSKOG JEZIKA}

U ovom radu analiziramo percepciju francuskih nazalnih vokala kod srpskih studenata francuskog jezika, vokala koji ne postoje u vokalskom sistemu srpskog jezika i koji često predstavljaju poteškoće u percepciji i produkciji učenicima/studentima čiji je srpski jezik maternji. Budući da se sistem percepcije formira tokom usvajanja maternjeg jezika, uho nije naviknuto da čuje foneme koje ne postoje u sistemu maternjeg jezika učenika što uzrokuje greške u percepciji tokom usvajanja glasova stranog jezika (Trubetzkoy [1938]2005; Best 1994; Kuhl 2000). Rezultati ovog istraživanja pokazuju da srpski studenti francuskog kao stranog jezika uspešno percipiraju i razlikuju oralnost od nazalnosti vokala dok imaju tendenciju da zamenjuju jedan nazalni vokal drugim. Najviše uspeha su

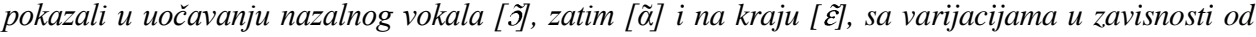
položaja vokala u reči ili ispitivane grupe studenata.

Ključne reči: nazalni vokali, percepcija, francuski jezik, srpski studenti, usvajanje 